\title{
A Study on Role of Ultrasonography in the Diagnosis of Dengue Fever
}

\author{
Y. Thathayya Naidu ${ }^{\oplus 1}$, R. Kiranmai ${ }^{\odot 2}$ \\ ${ }^{1}$ Assistant Professor, Department of Radio diagnosis, Konaseema Institute of Medical Sciences, Amalapuram, Andhra Pradesh, India, ${ }^{2}$ Assistant Professor, Department \\ of Radio diagnosis, Govt Medical College\& Hospital, Srikakulam, Andhra Pradesh, India.
}

\section{Abstract}

Background: Dengue Fever is an acute mosquito transmitted viral infection caused by one of the 4 serotypes of the genus flavivirus which has become a major international public health problem. The diagnosis of DF is often delayed owing to time taken for availability of serology test results. Moreover, this test is expensive and not widely available. Ultrasonography (USG) is a cheap, rapid and widely available non-invasive imaging method. In recent years several studies concluded that Ultrasonography of the chest and abdomen can be an important adjunct to clinical profile in diagnosis of DF and diagnosis can be made early in the course of the disease compared with other modes of diagnosis. The aim of the study is to demonstrate the ultrasound findings of dengue fever and also evaluate the specificity of gall bladder findings in dengue fever. Subjects and Methods: We conducted a prospective study in Govt Medical College\& Hospital, Srikakulam and Konaseema Institute of Medical Sciences, Amalapuram, A.P. Study included 50 patients referred to the department of Radio-Diagnosis and Imaging for Ultrasonography with clinical suspicion of dengue fever, during a period of July 2018 to December 2019. USG of the abdomen, pelvis and chest was performed in all cases and findings were noted. Dengue serology was performed later and all the ultrasound findings were correlated with dengue serology. Results: In our study of 50 patients all the patients studied were diagnosed with dengue fever based on dengue serology. In our study, $100 \%$ of our patients diagnosed with DF (by dengue serology) showed gall bladder wall thickening, $88 \%$ showed splenomegaly, $44 \%$ showed ascites. Pleural effusion was present in $30 \%$ of which $66.66 \%$ of pleural effusion was bilateral and the rest $33.33 \%$ was right sided. Isolated left sided pleural effusion was not found in our study. Hepatomegaly was present in $28 \%$ of our patients. In our study mortality and complications from dengue fever were not seen. Conclusion: Ultrasound findings in dengue fever are gall bladder wall thickening, splenomegaly, ascites, pleural effusion and hepatomegaly. In an area where DF is an epidemic, when Ultrasonography shows gall bladder wall thickening in a febrile patient with thrombocytopenia DF should be suggested On Ultrasonography, when there is gall bladder wall thickening, splenomegaly, ascites, and pleural effusion in a febrile patient with thrombocytopenia in a DF epidemic area a diagnosis of DF should be considered in a differential diagnosis until proved otherwise.

Keywords: Dengue Fever, Gall Bladder, Ascites, Splenomegaly, Hepatomegaly

Corresponding Author: R. Kiranmai, Assistant Professor, Department of Radio diagnosis, Govt Medical College\& Hospital, Srikakulam, Andhra Pradesh, India.

E-mail: rkiranmai2016@gmail.com

Received: 17 March 2020

Revised: 15 April 2020

Accepted: 26 April 2020

Published: 05 July 2020

\section{Introduction}

Dengue Fever is an acute mosquito-transmitted viral infection caused by 1 of 4 virus serotypes (DEN-1, DEN-2, DEN-3, and DEN-4) of the genus Flavivirus. It is the most common arboviral disease in the world. ${ }^{[1]}$ Dengue fever (DF) has been known for more than a century in the tropical areas of South East Asia and the Western Pacific regions. ${ }^{[2] ~ A ~ s i g n i f i c a n t ~}$ increase in the incidence of this infectious disease has taken place in the last 20 years and Dengue has become a major international public health concern in recent years. In 1998, it was deemed to be the most important tropical mosquitotransmitted infectious disease, surpassed only by malaria. ${ }^{[3]}$
It is now endemic in more than 100 countries and threatens the health of more than 2500 million i.e. $40 \%$ of the world's population. It is estimated that 10 million classic dengue infections occur each year with 500,000 cases of Dengue Hemorrhagic Fever. Its mortality ranges from $1-5 \%$ of treated patients to a maximum of $50 \%$ for untreated or poorly treated patients resulting in at least 12,000 deaths annually mainly among children. ${ }^{[4]}$

The disease occurs in two forms: classic dengue, the milder form of the disease and Dengue Hemorrhagic Fever (DHF), the severe form. The severity of this disease falls into four grades. Grade I, which is milder, is characterized by fever, general symptoms and positive tourniquet test and severe Grade IV 
which shows shock and non-detectable artery pressure. In all phases, there is thrombocytopenia and haemoconcentration. Since there is no other tests that can be used to diagnose the condition with a reasonable degree of accuracy and reliability, the diagnosis of DF is based on clinical appearance in combination with serology. Positive serology (anti dengue antibody) is the mainstay in the diagnosis of DF. ${ }^{[5]}$ But serology takes approximately 7 days to give a positive result. ${ }^{[6]}$ The diagnosis of DF is often delayed owing to time taken for availability of serology test results. Moreover, this test is expensive and not widely available. Ultrasonography (USG) is a cheap, rapid and widely available non- invasive imaging method. In recent years several studies concluded that ultrasonography of the chest and abdomen can be an important adjunct to clinical profile in diagnosis of DF and diagnosis can be made early in the course of the disease compared with other modes of diagnosis. ${ }^{[3]}$ It can be used as a first-line imaging modality in patients with suspected DF to detect early signs suggestive of the disease prior to obtaining serologic confirmation test results, especially in a DF epidemic area. ${ }^{[7]}$

Sonographic findings of DF have been described in several literatures. Some authors concluded that during an epidemic the ultrasound findings of gall bladder wall thickening with or without polyserositis in a febrile patient should suggest the possibility of DF/DHF. ${ }^{[4]}$ The reported changes vary according to the severity of each case. According to the previous studies ultrasound findings in milder form include pleural effusion, ascites, gall bladder wall thickening, hepatomegaly, splenomegaly, pancreatic enlargement, pericholecystic fluid and pericardial effusion. Severe form of the disease is characterized by fluid collection in the perirenal and pararenal region, hepatic and splenic subcapsular fluid collections, pericardial effusion, pleural effusions, ascites, gallbladder wall thickening, hepatomegaly, splenomegaly, hepatic intraparenchymal haemorrhages, and pancreatic enlargement. ${ }^{[3,4,7]}$

\section{Objectives}

To demonstrate the ultrasound findings of dengue fever.

To evaluate the specificity of gall bladder findings in dengue fever.

\section{Subjects and Methods}

\section{Source of data}

The study was conducted at Konaseema Institute of Medical Sceinces, Amalapuram \& Govt Medical College \& Hospital, Srikakulam, A.P. between July 2018-December 2019. 50 patients with clinical suspicion of DF referred to the Department Of Radio-diagnosis for Ultrasonography of abdomen and thorax were included in this study.

\section{Method of collection of data}

Prospective study of 50 patients.

\section{Inclusion criteria}

- Patients with signs and symptoms of DF such as fever, headache, myalgia, rashes, nausea, vomiting and abdominal pain.

- Patients of viral fever with thrombocytopenia.

- AGE GROUPS: all age groups

- SEX: Any

\section{Exclusion criteria}

- Patients in whom dengue serology was negative.

- Patients treated previously, for DF and currently asymptomatic.

\section{Equipment and protocol}

Ultrasonography of the abdomen was performed after 4-6hrs of fasting for better distention and visualisation of the gall bladder on USG machines Philips clear VUE 360, GE Logic F8. Gall bladder wall thickening was measured by placing the callipers between the two layers of anterior gall bladder wall. In all the cases gall bladder was assessed for wall thickening as well as for pericholecystic fluid collection and for presence of calculi. Gall bladder was imaged from the portal vein to fundus with transverse images obtained at representative images. Splenic and liver size was measured along the long axis from dome to the tip in a sagittal plane and the measurement of spleen more than $12 \mathrm{cms}$ and liver more than $15.5 \mathrm{cms}$ were considered as splenomegaly and hepatomegaly respectively. We examined the hepatorenal pouch and retrovesicular area in a supine position for ascites.

Thoracic Ultrasonography was done in supine or sitting position with assessment of both the pleural spaces in the entire posterior and lateral thorax. To detect pleural effusion, longitudinal scans of the right hemithorax at the midclaivular and the midaxillary line and a transverse scan of the right upper abdominal quadrant were performed in the supine position.

Dengue serology was performed on all suspected cases of DF and only those patients positive for DF were included in the study.

\section{Study design}

Hospital based prospective study

\section{Method of Statistical Analysis}

Excel software was used to analyze the statistical data.

\section{Results}

In our study, the highest number of cases was in the age group of 21-30yrs. The mean age was $37.7 \mathrm{yrs}$. The youngest patient was $12 \mathrm{yrs}$ and the oldest being $70 \mathrm{yrs}$. [Table 2] 


\begin{tabular}{lll}
\hline \multicolumn{2}{l}{ Table 1: Age Distribution } \\
\hline Age & Frequency & Percent \\
\hline $1-10 \mathrm{yrs}$ & 0 & 0 \\
$11-20 \mathrm{yrs}$ & 5 & 10 \\
$21-30 \mathrm{yrs}$ & 13 & 26 \\
$31-40 \mathrm{yrs}$ & 10 & 20 \\
$41-50 \mathrm{yrs}$ & 08 & 16 \\
$51-60 \mathrm{yrs}$ & 8 & 16 \\
$61-70 \mathrm{yrs}$ & 6 & 12 \\
\hline
\end{tabular}

Table 2: Mean Age of the patients

\begin{tabular}{llllll}
\hline N & Mean & Medial & SD & Min & Max \\
50 & 37.7 & 35 & 14.023 & 13 & 70 \\
\hline
\end{tabular}

Table 3: Gender frequency

\begin{tabular}{lll}
\hline Gender & Frequency & Percent \\
Male & 32 & 64 \\
Female & 18 & 36 \\
Total & 50 & 100 \\
\hline
\end{tabular}

Table 4: Distribution of study population according to clinical history

\begin{tabular}{lll}
\hline Clinical History & Frequency & Percent \\
Fever & 50 & 100 \\
Myalgia & 46 & 92 \\
Headache & 10 & 20 \\
Retro-Orbital Pain & 5 & 10 \\
\hline Vomiting & 5 & 10 \\
Abdominal Pain & 3 & 6 \\
\hline
\end{tabular}

Table 5: Distribution of rashes in study population

\begin{tabular}{lll}
\hline Rashes & Frequency & Percent \\
\hline Present & 30 & 60 \\
Absent & 20 & 40 \\
Total & 50 & 100 \\
\hline
\end{tabular}

In our study of 50 cases, 32(64\%) were males and 18(36\%) were females. [Table 3]

Among 50 cases, all (100\%) had fever and myalgia was present in $92 \%$. [Table 4]

Rashes were present in $60 \%$ of our cases. [Table 6]

In our study all the patients tested positive for dengue serology had gall bladder wall thickening, followed by ascites (44\%) and pleural effusion (30\%). [Table 7]

\begin{tabular}{|lll|}
\hline \multicolumn{3}{l}{ Table 6: Ultrasonography findings of study population. } \\
\hline Ultrasound Findings & Frequency & Percent \\
\hline $\begin{array}{l}\text { Gall Bladder Wall Thick- } \\
\text { ening (GBWT) }\end{array}$ & 50 & 100 \\
\hline Ascites & 22 & 44 \\
\hline Pleural Effusion & 15 & 30 \\
Hepatomegaly & 14 & 28 \\
\hline Splenomegaly & 44 & 88 \\
\hline
\end{tabular}

Table 7: Distribution of grades of ascites.

\begin{tabular}{lll}
\hline Ascites & Frequency & Percent \\
Moderate & 14 & 63.3 \\
Mild & 5 & 22.72 \\
Minimal & 3 & 13.63 \\
\hline
\end{tabular}

Table 8: Distribution of pleural effusion among patients

\begin{tabular}{lll}
\hline Pleural Effusion & Frequency & Percent \\
Bilateral & 10 & 66.66 \\
Right Sided & 5 & 33.33 \\
\hline
\end{tabular}

\begin{tabular}{lll}
\hline Table 9: diagnosis & & \\
\hline Diagnosis & Frequency & Percent \\
\hline DF & 36 & 72 \\
DHF & 14 & 28 \\
\hline
\end{tabular}

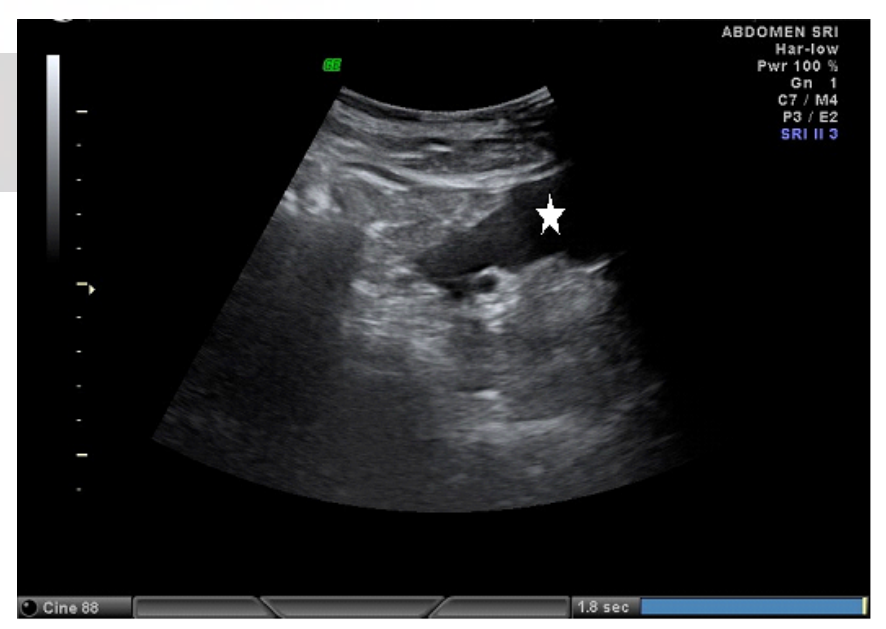

Figure 1: grey scale USG image of the pelvis showing anechoic free fluid in the pelvis suggestive of ascites.

Among 50 patients, ascites was present in $44 \%$ of our cases of which Moderate ascites was present in $63.63 \%$ of cases who had ascites. [Table 8] 


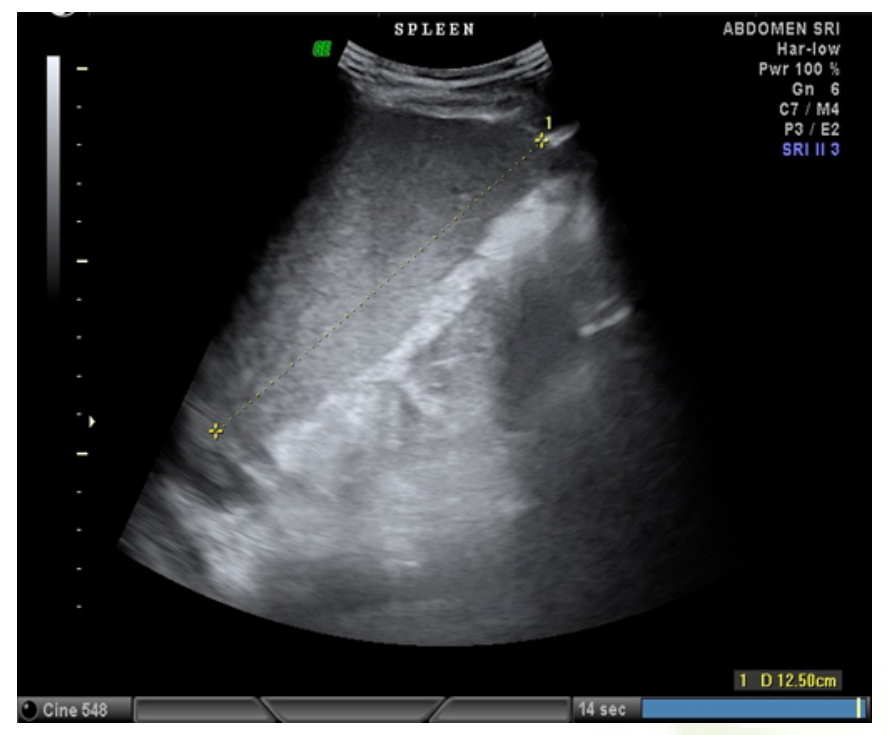

Figure 2: Grey scale USG image showing spleen measuring $12.5 \mathrm{cms}$ suggestive of splenomegaly.

In our study population of 50 patients, pleural effusion was present in $15(30 \%)$ of our cases, among whom $66.66 \%$ was bilateral and $33.33 \%$ was right sided. Isolated left sided pleural effusion was not present in our study. [Table 9]

In our study, DF was diagnosed in 72 percent of our cases and the rest 28 percent as DHF. [Table 10]

\section{Discussion}

Dengue is an acute febrile viral disease caused by flavivirus which occurs in two forms: DF, which is the milder form of the disease and the most severe DHF. Dengue has become a major international public health concern in recent years. DF is increasing due to uncontrolled population growth and improper urbanization in the absence of appropriate water management, global spread of dengue strains via travel and due to erosion of vector control programmes. In India the problem is even more acute because since 1963, more than 50 outbreaks have been reported by the National Institute of Communicable diseases, New Delhi.

Common laboratory findings include pancytopenia, neutropenia, increased haemoconcentration, thrombocytopenia and prolonged bleeding time. Serology is the mainstay in the diagnosis of DF. Haemagglutination inhibition antibodies usually appear at detectable level by day 5 to 6 of febrile illness. The diagnosis of DF is often delayed owing to time taken for availability of results. A diagnostic test that can quickly detect endothelial damage and facilitate immediate treatment is not currently available and the serological test needs paired serum samples with an interval of at least 7 days. Ultrasound can be used to detect small amounts of transudate in serous cavities in patients suspected of having DHF in endemic regions. Although not specific, the sonographic findings in DF are obtained more rapidly than the results of serologic tests. The early sonographic findings of DF have rarely been reported in the literature.

The aim of our study was to evaluate the ultrasound findings in DF, to find whether ultrasound of the abdomen is an important adjunct to clinical and laboratory profile in diagnosing DF and further if ultrasound is useful in predicting the severity of the disease.

Mia MW et al, ${ }^{[8]}$ conducted study on 100 patients as a part of clinical and sonological evaluation of DF and among these, $58 \%$ were males and $42 \%$ were females. In our study of 50 patients who were diagnosed as DF, 32(64\%) were male and $18(36 \%)$ were females. In our study DF was more common in males as compared to females which correlates with the study of Mia MW et al. ${ }^{[8]} T h$ ese findings are also consistent with findings of venkat sai $\mathrm{m}$ et al 4 that DF occurs more in male sex than females.

Keng liang wu 1 conducted study on 65 patients for early abdominal sonographic findings in patients with DF, of whom 36 were women and 29 were male. In their study they found DF more common in females than male which was the opposite in our case study.

Mia MW et al, ${ }^{[8]}$ conducted study on 100 patients as a part of clinical and sonological evaluation of DF and among these patients age ranged between 6-67years. In keng Liang wu et al 1 study of 65 patients the age ranged from 18-76 years with a mean age of 49 years as compared to our study the age range was from 12 to 70 years with a mean age of 37.7 years and DF was more prevalent in the age group between 21-30 years.

In Keng-Liang Wu et al, ${ }^{[1]}$ study on 65 patients, All 65 patients had fever. Thirty-three patients had skin rash, 35 had myalgia. Thirty-three patients had abdominal discomfort, and 24 patients experienced diarrhea. In Melani W. Setiawan et al, [9] study on 148 patients. The clinical features included fever in 148 (100\%), abdominal pain in 109 (74\%). Fever was also the main presenting complaint which was present in $100 \%$ of our study population, followed by myalgia $(92 \%)$, retroorbital pain $(10 \%)$, vomiting $(10 \%)$ and pain abdomen (6\%). In Mia MW et al, ${ }^{[8]}$ study fever was the most common clinical manifestation followed by nausea/vomiting and musculoskeletal pain. Rashes were present in $30(60 \%)$ of our study patients.

Skin rashes were present in $55 \%$ of our study patients which was also the case in a study performed by Keng-Liang Wu et al, ${ }^{[1]}$ study on 65 patients where Thirty-three patients had a skin rash.

The most common Ultrasonography feature in our study was gall bladder wall thickening which was present in all our study 
population making it 100\% which correlated with other studies conducted by P M Venkata Sai Et al, ${ }^{[4]}$ where gall bladder wall thickening was present in all of the dengue sero-positive cases. Thickened gall bladder wall was also the most common feature in studies conducted by Keng-Liang Wu et al, Melani W. Setiawan and Mia MW et al. ${ }^{[1,8,9]}$

The other features were Ascites which was present in $44 \%$ of our patients followed by pleural effusion in $30 \%$, splenomegaly in $88 \%$ and hepatomegaly in $28 \%$.

In Melani W. Setiawan study, ${ }^{[9]}$ Pleural effusion was found in $22 / 73(30 \%)$ of the cases. The pleural effusion was bilateral in 63/148 (43\%), right-sided in 30/148 (20\%), but never only on the left side as compared to our study Pleural effusion was present in 30\% and it was bilateral in and right sided in . left sided pleural effusion was not present in our study showing a good correlation between the two studies.

The above sonographic findings are not diagnostic of DF. Thickening of the gall bladder wall is also found in acute cholecystitis and some nonbiliary disorders namely hepatic cirrhosis, viral hepatitis, chronic congestive cardiac failure, chronic renal failure and hypoalbuminaemia. However, since gall bladder wall thickening was present in $100 \%$ of our cases it can be used as a supportive evidence to diagnose dengue fever prior to obtaining serological test which takes up to 7days to confirm dengue fever. Studies done by P M Venkata Sai Et $\mathrm{al},{ }^{[4]}$ concluded that diagnosis of DF can be made early in the course of disease based on Ultrasonography compared with other modes of diagnosis. During an epidemic the ultrasound findings of GB wall thickening with or without polyserositis in a febrile patient should suggest the possibility of DF/DHF.

Mortality and other complications from DF were not seen in our study probably due to early diagnosis and prompt treatment of DF and also milder form of the disease.

\section{Conclusion}

Ultrasound findings in dengue fever are gall bladder wall thickening, splenomegaly, ascites, pleural effusion and hepatomegaly. In an area where DF is an epidemic, when Ultrasonography shows gall bladder wall thickening in a febrile patient with thrombocytopenia DF should be suggested on Ultrasonography, when there is gall bladder wall thickening, splenomegaly, ascites, and pleural effusion in a febrile patient with thrombocytopenia in a DF epidemic area a diagnosis of DF should be considered in a differential diagnosis until proved otherwise.

\section{References}

1. Wu KL, Changchien CS, Kuo CH, Chiu KW, Lu SN, Kuo CM. Early abdominal sonographic findings in patients with dengue fever. J Clin Ultrasound. 2004;32(8):386-388. Available from: https://dx.doi.org/10.1002/jcu.20060.

2. Gubler DJ. Dengue and Dengue Hemorrhagic Fever. Clin Microbiol Rev. 1998;11(3):480-496.

3. Tusting LS, Bousema T, Smith DL, Drakeley C. Measuring changes in Plasmodium falciparum transmission: Precision, accuracy and costs of metrics. Adv Parasitol. 2014;84:151-208. Available from: https://dx.doi.org/10.1016/B978-0-12-800099$1.00003-\mathrm{X}$.

4. Sai PMV, Dev B, Krishnan R. Role of ultrasound in dengue fever. Brit J Radiol. 2005;78(929):416-418. Available from: https://dx.doi.org/10.1259/bjr/54704044.

5. Rahman M, Rahman K, Siddique AK, Shoma S, Kamal AHM, Ali KS. First outbreak of dengue haemorrhagic fever. Emerg Infect Dis. 2002;8(7):738-778. Available from: https://dx.doi. org/10.3201/eid0807.010398.

6. Parmar J, Mohan C, Kumar GP, Vora M. Ultrasound is Not Useful as a Screening Tool for Dengue Fever. Pol J Radiol. 2017;82:693-700. Available from: https://dx.doi.org/10.12659/ PJR.902861.

7. Wu KL, Changchien CS, Kuo CH, Chiu KW, Lu SN, Kuo CM, et al. Early abdominal sonographic findings in patients with dengue fever. Clin Ultrasound. 2004;32(8):386-394. Available from: https://doi.org/10.1002/jcu.20060.

8. Mia MW, Nurullah AM, Hossan A, Haque M. Clinical and sonological evaluation of dengue fever in Bangladesh. Dinajpur Med Coll J. 2003;(1):29-34.

9. Setiawan MW, Samsi TK, Wulur H, Sugianto D, Pool TN. Dengue haemorrhagic fever: ultrasound as an aid to predict the severity of the disease. Pediat Radiol. 1998;28(1):1-4. Available from: https://dx.doi.org/10.1007/s002470050281.

Copyright: (C) the author(s), 2020. It is an open-access article distributed under the terms of the Creative Commons Attribution License (CC BY 4.0), which permits authors to retain ownership of the copyright for their content, and allow anyone to download, reuse, reprint, modify, distribute and/or copy the content as long as the original authors and source are cited.

How to cite this article: Naidu YT, Kiranmai R. A Study on Role of Ultrasonography in the Diagnosis of Dengue Fever. Asian J. Med. Radiol. Res. 2020;8(1):139-143.

DOI: dx.doi.org/10.47009/ajmrr.2020.8.1.25

Source of Support: Nil, Conflict of Interest: None declared. 